\title{
The search for the neutron electric dipole moment at PSI
}

\author{
G. Pignol $^{1 \star}$ and P. Schmidt-Wellenburg ${ }^{2 \star}$ on behalf of the nEDM collaboration \\ 1 Université Grenoble Alpes, Centre National de la Recherche Scientifique, Grenoble INP, \\ LPSC-IN2P3, Grenoble, France \\ 2 Paul Scherrer Institute, 5232 Villigen, Switzerland \\ *philipp.schmidt-wellenburg@psi.ch
}

February 2, 2021

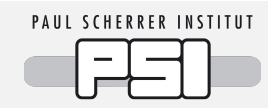

Review of Particle Physics at PSI

doi:10.21468/SciPostPhysProc.2

\section{Abstract}

\begin{abstract}
The existence of a nonzero permanent electric dipole moment (EDM) of the neutron would reveal a new source of CP violation and shed light on the origin of the matterantimatter asymmetry of the Universe. The sensitivity of current experiments using stored ultracold neutrons (UCN) probes new physics beyond the TeV scale. Using the UCN source at the Paul Scherrer Institut, the nEDM collaboration has performed the most sensitive measurement of the neutron EDM to date, still compatible with zero $\left(\left|d_{n}\right|<1.8 \times 10^{-26} e \mathrm{~cm}\right)$. A new experiment designed to improve the sensitivity by an order of magnitude, n2EDM, is currently in construction.
\end{abstract}

\subsection{Introduction}

The permanent electric dipole moment (EDM) $d$ of a simple quantum system of spin $1 / 2$ represents the coupling between the particle spin and an externally applied electric field $\vec{E}$, in the same way that the magnetic dipole moment $\mu$ quantifies the coupling between the spin and an applied magnetic field $\vec{B}$. The spin dynamics is entirely described by the Hamiltonian

$$
\hat{H}=-\mu \hat{\vec{\sigma}} \cdot \vec{B}-d \hat{\vec{\sigma}} \cdot \vec{E},
$$

where $\vec{\sigma}$ are the Pauli matrices. Because $\overrightarrow{\vec{\sigma}} \cdot \vec{E}$ is odd with respect to time reversal, the CPT theorem implies that a non-zero EDM would result in a violation of CP symmetry. The search for a nonzero EDM was initiated in the 1950's [1], applying the newly invented resonance method with separated oscillating fields [2] on a thermal neutron beam. The quest for an EDM was then extended to many other systems, as shown in Figure 27.1, (see [3] for a review on EDM searches). All experiments to date have reported results compatible with zero, despite the million-fold improvement of the sensitivity of modern experiments. As discussed in the theory chapter of this volume, the present limits on EDMs provide stringent constraints on theories beyond the Standard Model of particle physics, which generally predict new sources of CP violation and therefore non-zero EDMs. The next generation of experiments with improved sensitivity are motivated by the exciting possibility of discovering a non-zero EDM induced by new physics at the multi-TeV scale.

An international collaboration of 15 laboratories (the $n E D M$ collaboration) is conducting a long-term program at PSI to search for the neutron EDM. In 2009, the RAL/Sussex/ ILL instrument [5], which was previously used at the Institut Laue Langevin in Grenoble for a long series of nEDM measurements [6-9], was connected to the newly built high-intensity 


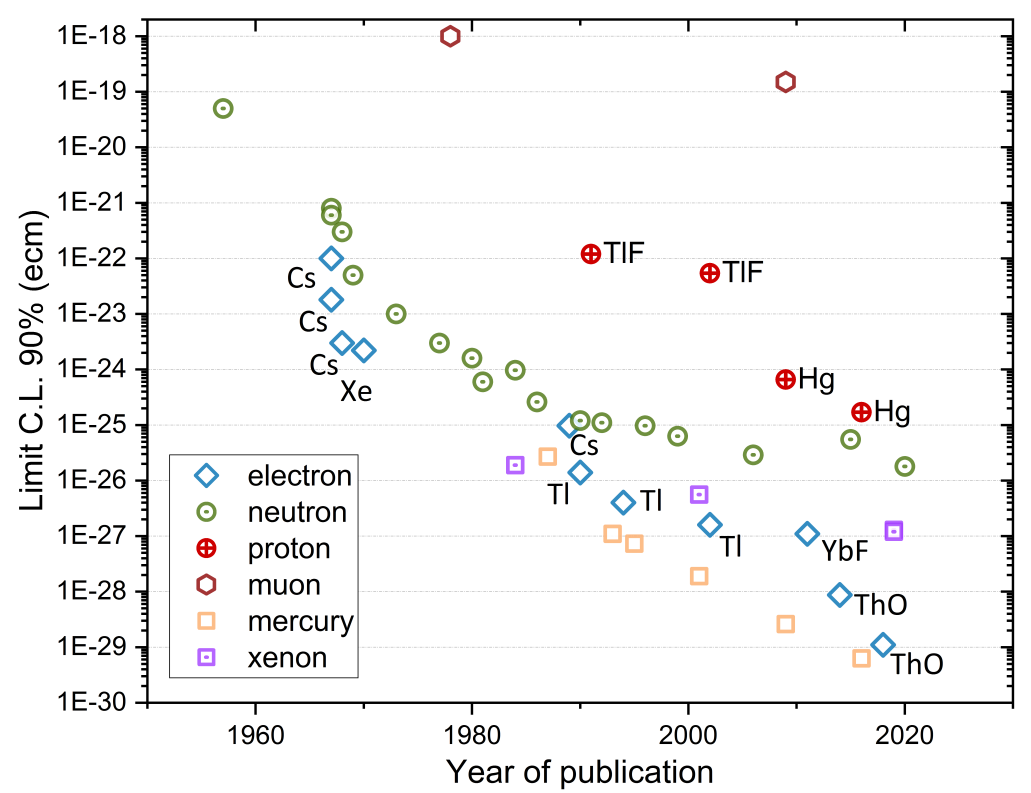

Figure 27.1: History of upper limits (90 \% C.L.) for the EDM of various systems. Image first published in [4].

source of ultracold neutrons $[10,11]$. After a phase of hardware upgrades and commissioning of the instrument, data was collected during 2015 and 2016. This resulted in the currently most precise measurement of the neutron EDM, $d_{\mathrm{n}}=\left(0.0 \pm 1.1_{\text {stat }} \pm 0.2_{\text {sys }}\right) \times 10^{-26} e \cdot \mathrm{cm} \mathrm{[12]}$. This measurement, with the single chamber instrument, will be described in Section 27.3. The construction of the new double chamber instrument (called n2EDM: the new neutron EDM apparatus) started in 2018. It will be described in Section 27.4. In the next section we elaborate on the main challenges to neutron EDM searches.

\subsection{The three challenges for searches for the neutron EDM}

The coupling in (27.1) leads to a precession of the neutron spin around the fields at an angular frequency given by $\omega=2(\mu B+d E) / \hbar$ in parallel electric and magnetic fields. In principle the EDM term can be separated from the magnetic term by taking the difference of the frequency measured in parallel and anti-parallel field configurations. However, the electric term that is to be measured is extremely small. For $d=10^{-26} \mathrm{ecm}$ and $E=15 \mathrm{kV} / \mathrm{cm}$, the spin would complete just about two full turns per year, due to the electric term. For the detection of such a minuscule coupling, one needs (i) a long interaction time with a large electric field, (ii) a high flux of neutrons, and (iii) precise control of the magnetic field. These requirements constitute the three main challenges for the measurement.

The neutron precession frequency is measured using Ramsey's resonance method: neutrons with spins parallel to the magnetic field are selected, then a first oscillating transverse magnetic-field pulse is applied with a strength and duration adjusted to tilt the spin into the plane transverse to the magnetic field. The spins then precess freely during a precession time $T$, after which a second pulse, identical to and in phase with the first one, is applied. At the end of the process the neutron spins are analyzed in order to extract the asymmetry $A$ of neutrons counted with spin up and down. The asymmetry is a function of the applied pulse frequency and of the precession frequency to be measured, as shown in Figure 27.2. By measuring the asymmetry, the neutron precession frequency $f_{n}$ is extracted. After combining several measurements, aka cycles, of $f_{n}$ with different polarities of the electric field the neutron EDM is 


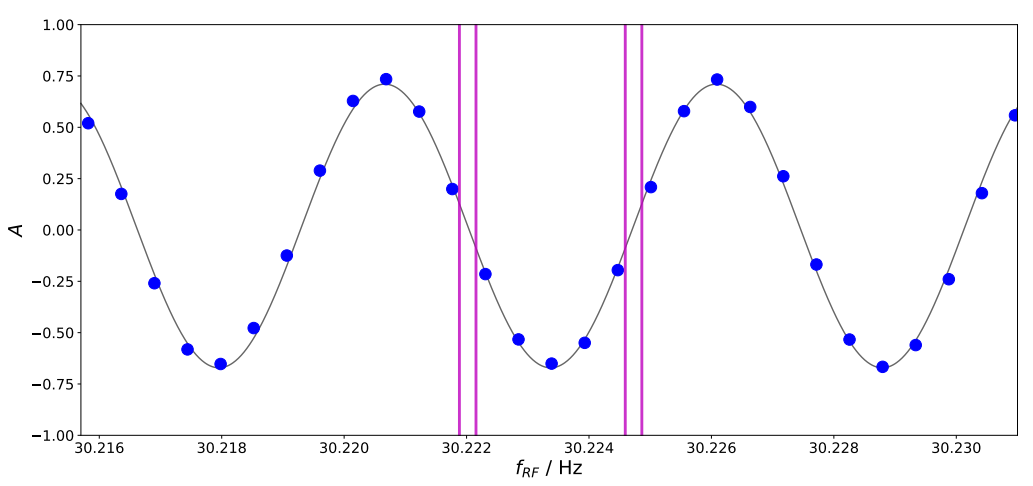

Figure 27.2: Measurement of the asymmetry $A=\left(N_{\uparrow}-N_{\downarrow}\right) /\left(N_{\uparrow}+N_{\downarrow}\right)$ as a function of the applied frequency $f_{\mathrm{RF}}$ of the pulses. Each point is a measurement cycle with a precession time of $T=180$ s performed with the single chamber apparatus in 2017. The vertical bars show the position of the four "working points" used in the nEDM data-taking to maximize the sensitivity. The line is a fit of (27.3) to the data.

measured with a statistical sensitivity per cycle of

$$
\sigma\left(d_{n}\right)=\frac{\hbar}{2 E T \alpha \sqrt{N}},
$$

where $N$ is the total number of neutron counts and $\alpha$ is the visibility of the resonance corresponding to the ensemble polarization of the neutrons at the end of the precession period. It is apparent from (27.2) that the combination $E T$ enters linearly in the statistical sensitivity and must be maximized (first challenge) along with the statistical factor $\sqrt{N}$ (second challenge).

The first neutron EDM experiments used beams of neutrons interacting with the fields for only a few milliseconds. The turning point for higher sensitivities was the advent of ultracold neutron (UCN) sources which permitted neutrons to be stored in a precession chamber for a duration approaching the neutron half-life of 10 minutes. Care must be taken in the choice of materials constituting the precession chamber in order to minimize neutron losses.

In the single chamber apparatus, the precession chamber was a cylinder of radius $23.5 \mathrm{~cm}$ and height $12 \mathrm{~cm}$, assembled from two aluminum electrodes coated with diamond-like-carbon [13-16] and a polystyrene ring coated with deuterated polystyrene [17]. In average $N=15000$ neutrons per cycle were exposed to an electric field of $11 \mathrm{kV} / \mathrm{cm}$ during $T=180 \mathrm{~s}$.

Based on experience and demonstrated developments, a double chamber apparatus was designed. Two vertically stacked chambers, with larger radii of $40 \mathrm{~cm}$ will sustain a larger electric field of opposite polarity and store more neutrons.

Table 27.1 shows the main parameters determining the statistical sensitivity.

\begin{tabular}{c|c|c} 
& single chamber (2016) & double chamber (projection) \\
\hline \hline$N$ (per cycle) & $15^{\prime} 000$ & $121^{\prime} 000$ \\
$T$ & $180 \mathrm{~s}$ & $180 \mathrm{~s}$ \\
$E$ & $11 \mathrm{kV} / \mathrm{cm}$ & $15 \mathrm{kV} / \mathrm{cm}$ \\
$\alpha$ & 0.75 & 0.8 \\
\hline$\sigma\left(d_{n}\right)$ per day & $11 \times 10^{-26} \mathrm{e} \cdot \mathrm{cm}$ & $2.6 \times 10^{-26} \mathrm{e} \cdot \mathrm{cm}$ \\
\hline \hline
\end{tabular}

Table 27.1: Comparison between (i) the achieved performance of the single chamber apparatus during the datataking at PSI in 2016, (ii) nominal parameters for the design of n2EDM. 


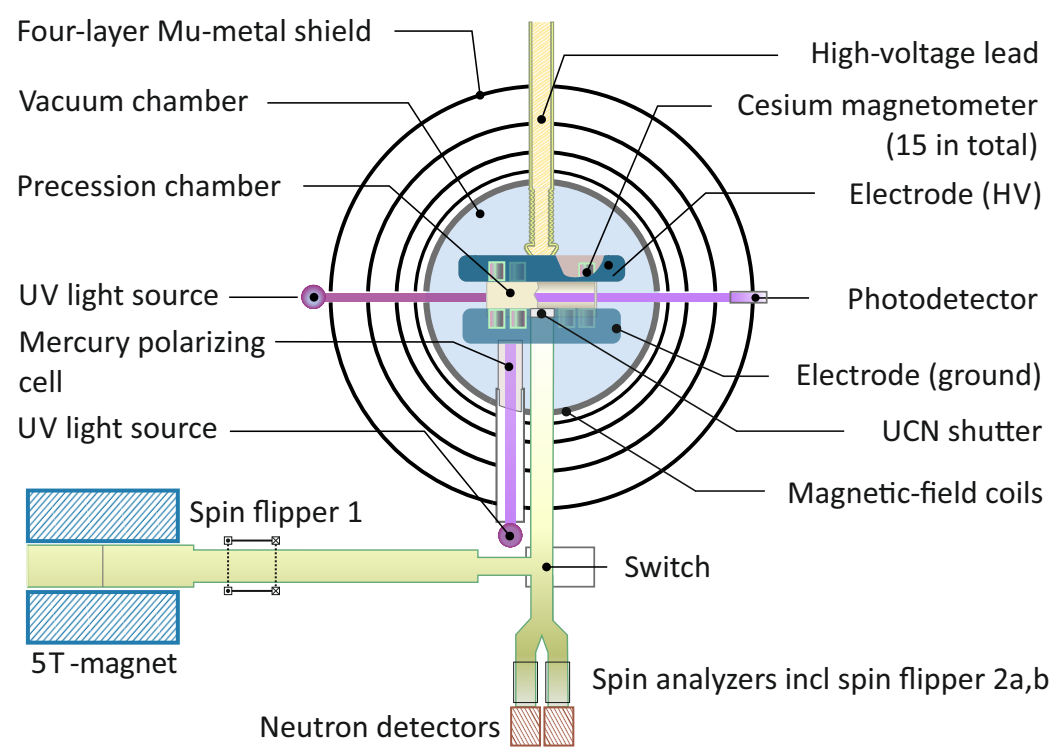

Figure 27.3: Scheme of the single chamber experiment operated during 2009-2017 at PSI. Image first published in [?].

The high statistical sensitivity must be combined with precise control of the magnetic field: the third challenge. This is accomplished with a combination of magnetic shielding, the generation of a stable and uniform magnetic field inside the shield, and measurements of the magnetic field with atomic magnetometry. In the single chamber experiment, the change of the magnetic field between reversals of the electric polarity (typically every 4 hours), needed to be controlled at a level better than $10 \mathrm{fT}$. This was established by making sure that the Allan deviation for a field average over 4 hours was below $10 \mathrm{fT}$.

For this purpose, the co-magnetometer technique [18,19] was used. Polarized ${ }^{199} \mathrm{Hg}$ atoms were injected in the chamber and the precession frequency of the atoms was measured optically, providing the magnetic-field average over the same time and almost the same volume as the neutrons.

The mercury co-magnetometer is essential to control the residual time variations of the magnetic field (both correlated and uncorrelated with the electric polarity). However, this comes at the price of inducing a false EDM due to the combined effect of the relativistic motional field $v \times E / c^{2}$ seen by the mercury atoms and the magnetic field non-uniformities [20-23]. Due to this important systematic effect, the control of the uniformity of the magnetic field is of utmost importance. In particular, ferromagnetic impurities close to the precession chamber(s) must be avoided, and the residual large-scale magnetic gradients must be minimized and measured with a combination of online and offline methods.

\subsection{Measurement and result}

The principal characteristic of the instrument operated between 2009 to 2017 at PSI was a single-chamber precession volume for UCN, which at the same time contained spin-polarized ${ }^{199} \mathrm{Hg}$ atoms as reference or cohabiting magnetometer [18, 19].

Figure 27.3 shows a technical sketch of the instrument. Ultracold neutrons from the PSI UCN source $[11,24]$ were polarized upon the passage through the $5 \mathrm{~T}$ solenoid and entered the precession chamber from the bottom. The spin-manipulation and free precession of UCN and ${ }^{199} \mathrm{Hg}$ took part here, $125 \mathrm{~cm}$ above the horizontal beam line, inside a 4-layer mu-metal 
shield. The top electrode made contact to the tip of a high voltage (HV) feed-through tested in operation up to $200 \mathrm{kV}$. An electric field of $E= \pm 11 \mathrm{kV} / \mathrm{cm}$ was used for data-taking. The magnetic field, $B \approx 1 \mu \mathrm{T}$, was generated by a current of about $17 \mathrm{~mA}$ in a $\cos \theta$-coil wound directly onto the cylindrical vacuum tank. In addition to the $\cos \theta$-coil there were a total of 35 saddle and cylindrical coils, aka trim coils, wound on the tank to adjust magneticfield gradients. Two of these saddle coils, on the top and bottom of the vacuum tank, were used to set a small vertical magnetic-field gradient $\partial B_{z} / \partial z$, for each sequence. The ${ }^{199} \mathrm{Hg}$ comagnetometer measured the time and volume averaged magnetic field within the precession chamber and was subject to the above-described motional systematic effect. At the same time an array of 15 optically-pumped Cs vapor magnetometers (CsM) [25], mounted above and below the chamber, was used to monitor the magnetic-field uniformity with a sampling rate of $1 \mathrm{~Hz}$. Another three coils, two of them in a Helmholtz-like geometry and one a saddle coil, wound onto the outside of the vacuum tank were used to generate the spin-manipulation pulses, once the UCN and ${ }^{199} \mathrm{Hg}$-atoms were inside the chamber, with frequencies close to the resonance Larmor frequency of ${ }^{199} \mathrm{Hg}(\sim 7.8 \mathrm{~Hz})$ and neutron $(\sim 30.2 \mathrm{~Hz})$.

After the second $t=2 \mathrm{~s}$ long spin-flip pulse of the Ramsey sequence the neutrons were counted in a spin-sensitive detection system $[26,27]$. For each cycle, from the recorded number of neutrons with spin up $N_{u}$ and down $N_{d}$ the asymmetry $A_{i}=\left(N_{u, i}-N_{d, i}\right) /\left(N_{u, i}+N_{d, i}\right)$ was computed. During data taking, the files containing the detector data were blinded by injection of an artificial unknown EDM signal [28], different for two distinct analysis groups.

During the nEDM data acquisition period from July 2015 until December 2016 a total of 54068 cycles each with an average of about 11400 neutrons were recorded. The data were taken with different magnetic-field configurations, e.g. $B$ up or downwards pointing with $-25 \mathrm{pT} / \mathrm{cm} \geq \partial B_{z} / \partial z \leq 25 \mathrm{pT} / \mathrm{cm}$. Each of these sequences contained several hundred cycles and multiple electric-field changes as can be seen in Figure 27.4. A total of 99 sequences were analyzed. In a first step, each sequence was divided into sub-sequences including at least two changes of the electric field polarity. The data of a sub-sequence, typically 114 cycles, was fit to

$$
A_{i}=A_{\text {off }} \mp \alpha \cos \left(\frac{\pi f_{\mathrm{rf}}^{\prime}}{v}+\phi\right),
$$

where $f_{\mathrm{rf}}^{\prime}$ is the neutron spin flip frequency corrected for magnetic-field drift using the measured $f_{\mathrm{Hg}}$ and $v=1 /(T+4 t / \pi)$ is the width (FWHM) of the central fringe (see Figure 27.2). To extract the neutron resonance frequency, $f_{\mathrm{n}, i}$, the fit parameters $A_{\text {off }}, \alpha$ were fixed for each cycle and (27.3) was solved for $\phi=\pi f_{\mathrm{n}, i} / v$. Figure 27.4 bottom shows the ratio $\mathcal{R}_{i}=f_{\mathrm{n}, i} / f_{\mathrm{Hg}, i}$ for a full measurement sequence. An optimized analysis strategy was implemented, accounting for all known effects [12] which affect the $\mathcal{R}$ ratio:

$$
\begin{aligned}
\mathcal{R}=\left|\frac{\gamma_{n}}{\gamma_{\mathrm{Hg}}}\right| & \left(1+\delta_{\mathrm{EDM}}+\delta_{\mathrm{EDM}}^{\text {false }}+\delta_{\text {quad }}\right. \\
& \left.+\delta_{\text {grav }}+\delta_{\mathrm{T}}+\delta_{\text {Earth }}+\delta_{\text {light }}+\delta_{\text {inc }}+\delta_{\text {other }}\right),
\end{aligned}
$$

in particular the EDM term $\delta_{\mathrm{EDM}}=2 E /\left(\hbar \gamma_{n} B\right) d_{n}$. In fact, the dominating effect is the gravitational shift $\delta_{\text {grav }}=G_{\text {grav }}\langle z\rangle / B$, which is due to the relative center-of-mass offset $\langle z\rangle=-0.39(3) \mathrm{cm}$ between UCN and ${ }^{199} \mathrm{Hg}$. This is both a source of drifts (a nuisance) and also an excellent measure of the effective vertical magnetic-field gradient $G_{\text {grav }}$. In each sub-sequence, the EDM signal $d_{n}^{\text {meas }}$ and $\langle\mathcal{R}\rangle$ are determined by fitting the $\mathcal{R}_{i}$ values, compensated for the relative gradient drift, as a function of time and electric field by allowing, also, for a linear time drift, as shown in Figure 27.5. The measured $d_{n}^{\text {meas }}$ for a given field configuration is shifted by the term $\delta_{\mathrm{EDM}}^{\mathrm{false}}=2 E /\left(\hbar \gamma_{n} B\right) d^{\text {false }}$ corresponding to the motional false effect of ${ }^{199} \mathrm{Hg}$ mentioned 

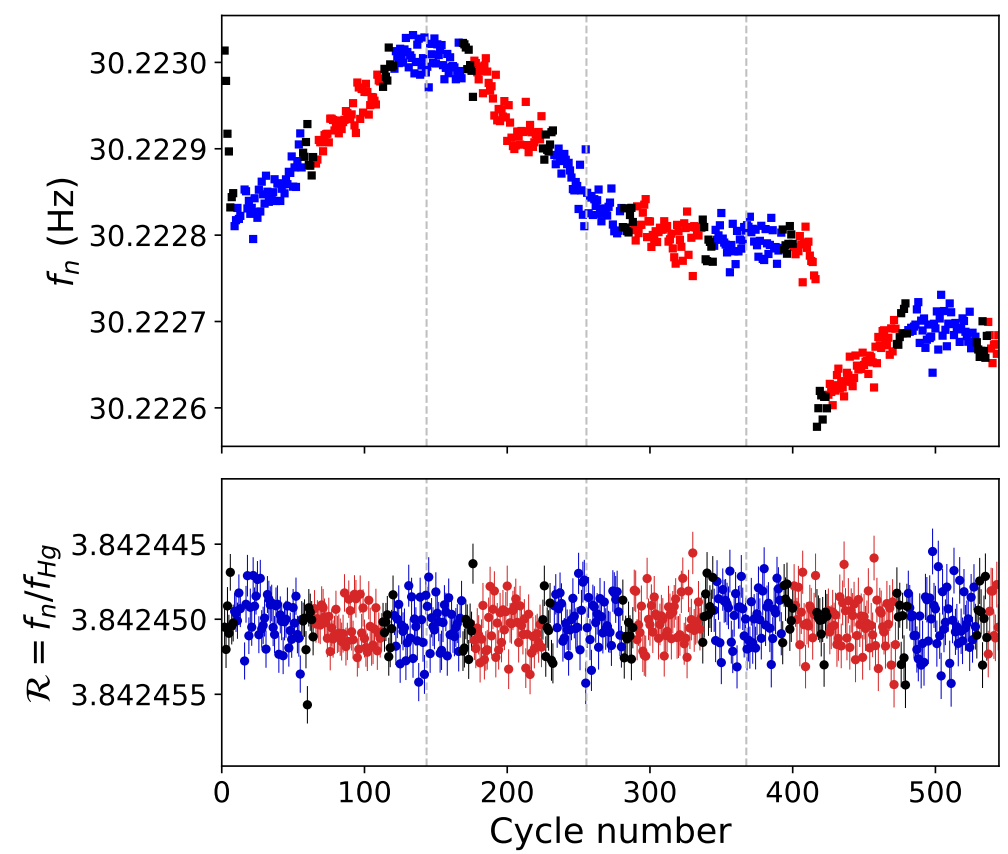

Figure 27.4: Plot of neutron frequency (top), $f_{n}$, and frequency ratio (bottom), $\mathcal{R}$, for a full sequence of nEDM data. Red data points indicate a positive voltage, while negative are marked blue. Black is used for cycles without electric field. A single EDM value is extracted for each sub-sequence, indicated by vertical dashed lines, before a weighted EDM average is calculated for the entire sequence. Figure reused from [?].

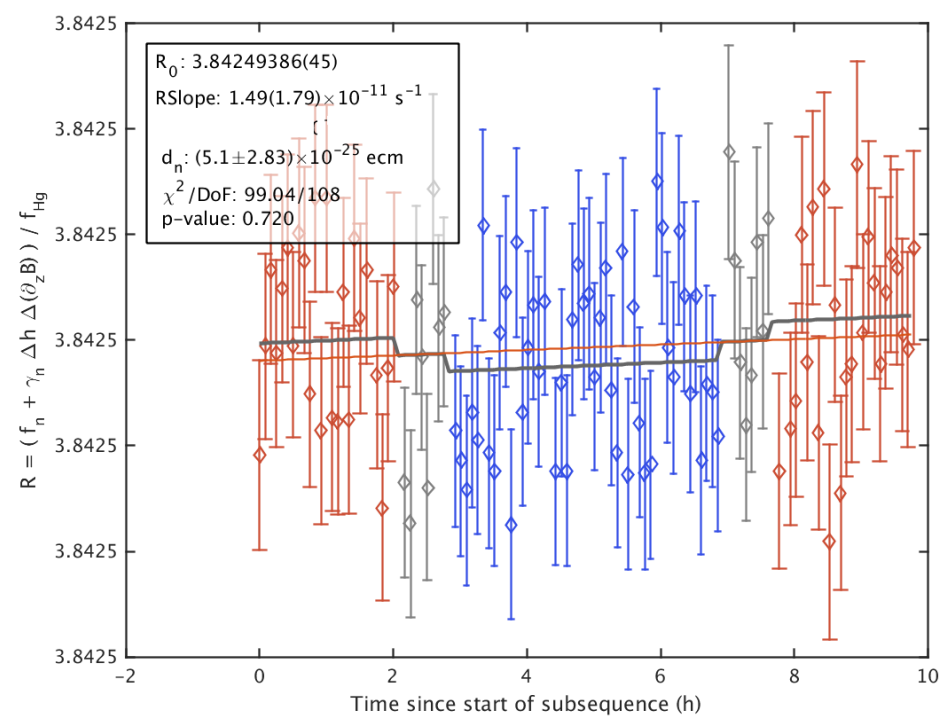

Figure 27.5: Subsequence with two polarity changes and a linear fit in time and $d_{n} E$ offsets. Note, that $E=-U / d$ hence positive electric fields (red) result from a negative charged electrode in Figure 27.4. 
previous section. This effect depends on the magnetic field gradients and can be expressed as $[25]$ :

$$
d^{\text {false }}=\frac{\hbar}{8 c^{2}}\left|\gamma_{n} \gamma_{\mathrm{Hg}}\right| R^{2}\left(G_{\text {grav }}+\hat{G}\right),
$$

where $\hat{G}$ is the contribution from higher-order gradients and does not produce a gravitational shift. After correction of $\langle\mathcal{R}\rangle$ and $d_{n}^{\text {meas }}$ for $\delta_{\mathrm{T}}$ and $\delta_{\text {Earth }}$, the contribution from $\hat{G}$, and minor systematic shifts, the remaining shift is linear in $G_{\text {grav }}$ and was removed by a crossing point fit as shown in Figure 4 of [12]. The results of the crossing-point fit after unblinding of the two analysis teams were $d_{\times, 1}=(-0.09 \pm 1.03) \times 10^{-26} e \cdot \mathrm{cm}, \mathcal{R}_{\times, 1}=3.8424546$ (34) with $\chi^{2} /$ dof $=106 / 97$ and $d_{\times, 2}=(0.15 \pm 1.07) \times 10^{-26} e \cdot \mathrm{cm}, \mathcal{R}_{\times, 2}=3.8424538$ (35) with $\chi^{2} /$ dof $=105 / 97$. The excellent agreement of both $\mathcal{R}_{\times}$values with each other and with the literature value $\gamma_{n} / \gamma_{\mathrm{Hg}}=3.8424574(30)$ [23], demonstrates the excellent control and understanding of all magnetic-field-related shifts [25].

\section{4 n2EDM: The double chamber apparatus}

The concept and design of the new double chamber instrument, n2EDM [29], was based on maximizing the statistical sensitivity of a single measurement, see Table 27.1, while at the same time further reducing systematic effects.

As can be seen in Figure 27.6, the new apparatus has two cylindrical storage chambers of diameter $\varnothing 80 \mathrm{~cm}$, made from proven materials, stacked one above the other, separated only by a common high voltage electrode in the center. The UCN transport and storage layout was optimized for a maximum number of neutrons per cycle using the established and bench marked Monte Carlo code of the collaboration [30]. This resulted in ultracold-neutron guides with constant effective cross section and sub-nanometer roughness along the path up to the two precession chambers which in turn are placed at the optimal height relative to the beam line.

Both chambers are centered inside the same uniform magnetic field generated by a main magnetic-field coil and an advanced trim-coil system within a 6-layer magnetic and one-layer Eddy current shield. First measurements of the quasi-static shielding factor in 2020 exceeded the specified value of 80000 in all directions. This is supplemented by an active magnetic shield (AMS), similar to the active coil system used previously [31], with eight degrees of freedom devised to further improve the shielding factors at very low frequencies. Dedicated coils were designed [32] and mounted onto the inner wall surfaces of the wooden thermal enclosure to compensate gradient magnetic fields up to first order. Hence, neutrons and mercury inside the two precession chambers are exposed to the same extremely low noise, highly uniform magnetic field while the electric field points in opposite directions. We expect that an application of electric fields up to $|E| \geq 15 \mathrm{kV} / \mathrm{cm}$ can be achieved without difficulties, as the HV electrode is entirely enclosed in a grounded Faraday cage.

All CsM are placed at ground potential and the previous limitation on the electric-field strength due to flashovers along optical fibers of the CsM can be ruled out. The sensors were designed for an operation in Bell-Bloom mode [33], recording free spin-precession waveforms for highest accuracy and with a sensitivity of better than $200 \mathrm{fT} / \sqrt{\mathrm{Hz}}$. This is an essential improvement for the accurate determination of higher order magnetic-field terms relevant for the correction of systematic effects.

Each precession chamber is connected via a UCN switch to a simultaneous spin detection device featuring each two UCN detectors. A gas mixture of $\mathrm{CF} 4$ and ${ }^{3} \mathrm{He}$ is used for neutron detection. The short scintillation pulse is registered by large surface photo-multipliers and enables high count rate with very low background counts from gamma rays or cosmic radiation.

In summary the new double chamber spectrometer, n2EDM, at PSI combines the newest concepts and technologies while relying on proven techniques and methods to improve the 


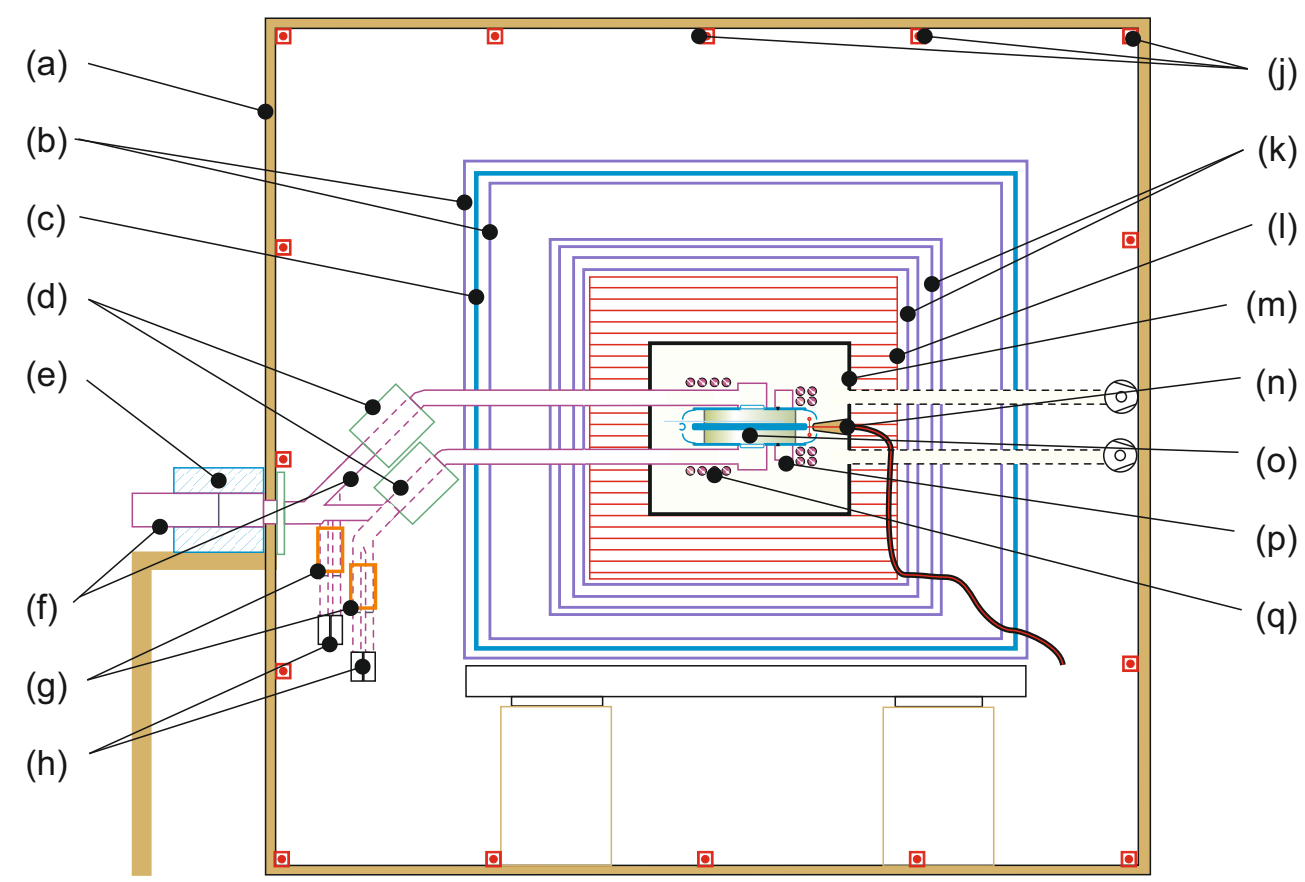

Figure 27.6: Sketch of the new double chamber instrument "n2EDM" at PSI from [29]. (a) Thermal shell, (b) outer MSR shell, (c) Eddy current shield, (d) UCN switches, (e) 5 T-solenoid, (f) UCN guides, (g) fast adiabatic spin flippers, (h) UCN detectors, (j) AMS, (k) inner MSR shells, (l) magnetic field coils, (m) vacuum chamber connected to turbo pumps, (n) high voltage feed through and cable, (o) double precession chamber with central electrode, (p) ${ }^{199}$ Hgpolarization cell, (q) cesium magnetometers.

sensitivity frontier.

An attractive future option, which is described in great detail in [34], eliminates the motional false EDM by adjusting the magnetic-field strength so that the integral in equation (9) in [29] vanishes. This magic field configuration indicates a possible path to ultimate sensitivity using the n2EDM spectrometer at PSI.

\subsection{Outlook and world-wide competition}

With the publication of the latest, most stringent limit of $d_{\mathrm{n}}<1.8 \times 10^{-26} \mathrm{e} \cdot \mathrm{cm}$, PSI became the fourth member of the exclusive club of institutes that have hosted a successful nEDM search. It is now competing with a group of fierce and passionate competitors from all around the world [35-39] to break into the range of $1 \times 10^{-27} \mathrm{e} \cdot \mathrm{cm}$ within the next decade. A discovery of an nEDM or a further improved limit would markedly and indelibly shape future models of particle physics beyond the current Standard Model.

\section{References}

[1] J. H. Smith, E. M. Purcell and N. F. Ramsey, Experimental Limit to the Electric Dipole Moment of the Neutron, Phys. Rev. 108, 120 (1957), doi:10.1103/PhysRev.108.120.

[2] N. F. Ramsey, A Molecular Beam Resonance Method with Separated Oscillating Fields, Phys. Rev. 78(6), 695 (1950). 
[3] T. E. Chupp, P. Fierlinger, M. J. Ramsey-Musolf and J. T. Singh, Electric dipole moments of atoms, molecules, nuclei, and particles, Reviews of Modern Physics 91(1), 015001 (2019), doi:10.1103/RevModPhys.91.015001.

[4] K. Kirch and P. Schmidt-Wellenburg, Search for electric dipole moments, EPJ Web Conf. 234, 01007 (2020), doi:10.1051/epjconf/202023401007, 2003.00717.

[5] C. Baker et al., Apparatus for Measurement of the Electric Dipole Moment of the Neutron using a Cohabiting Atomic-Mercury Magnetometer, Nucl. Instrum. Meth. A 736, 184 (2014), doi:10.1016/j.nima.2013.10.005, 1305.7336.

[6] J. Pendlebury, K. Smith, R. Golub, J. Byrne, T. McComb, T. Sumner, S. Burnett, A. Taylor, B. Heckel, N. Ramsey, K. Green, J. Morse et al., Search for a neutron electric dipole moment, Phys. Lett. B 136(5-6), 327 (1984).

[7] K. F. Smith, N. Crampin, J. M. Pendlebury, D. J. Richardson, D. Shiers, K. Green, A. I. Kilvington, J. Moir, H. B. Prosper, D. Thompson, N. F. Ramsey, B. R. Heckel et al., A search for the electric dipole moment of the neutron, Phys. Lett. B 234, 191 (1990), doi:10.1016/0370-2693(90)92027-G.

[8] P. Harris et al., New experimental limit on the electric dipole moment of the neutron, Phys. Rev. Lett. 82, 904 (1999), doi:10.1103/PhysRevLett.82.904.

[9] C. Baker et al., An Improved experimental limit on the electric dipole moment of the neutron, Phys. Rev. Lett. 97, 131801 (2006), doi:10.1103/PhysRevLett.97.131801, hep-ex/0602020.

[10] A. Anghel, F. Atchison, B. Blau, B. van den Brandt, M. Daum, R. Doelling, M. Dubs, P.A. Duperrex, A. Fuchs, D. George, L. Göltl, P. Hautle et al., The PSI ultra-cold neutron source, Nucl. Instrum. Methods A 611(2-3), 272 (2009).

[11] B. Lauss, Ultracold Neutron Production at the Second Spallation Target of the Paul Scherrer Institute, Phys. Proc. 51, 98 (2014).

[12] C. Abel et al., Measurement of the permanent electric dipole moment of the neutron, Phys. Rev. Lett. 124(8), 081803 (2020), doi:10.1103/PhysRevLett.124.081803, 2001.11966.

[13] F. Atchison et al., First storage of ultracold neutrons using foils coated with diamond-like carbon, Phys. Lett. B 625, 19 (2005), doi:10.1016/j.physletb.2005.08.066.

[14] F. Atchison et al., Diamondlike carbon can replace beryllium in physics with ultracold neutrons, Phys. Lett. B 642, 24 (2006), doi:10.1016/j.physletb.2006.09.024.

[15] F. Atchison et al., Storage of ultracold neutrons in a volume coated with diamondlike carbon, Phys. Rev. C 74, 055501 (2006), doi:10.1103/PhysRevC.74.055501.

[16] F. Atchison et al., Loss and spinflip probabilities for ultracold neutrons interacting with diamondlike carbon and beryllium surfaces, Phys. Rev. C 76, 044001 (2007), doi:10.1103/PhysRevC.76.044001.

[17] K. Bodek, M. Daum, R. Henneck, S. Heule, M. Kasprzak, K. Kirch, A. Knecht, M. Kuźniak, B. Lauss, M. Meier, G. Petzoldt, M. Schneider et al., Storage of ultracold neutrons in high resistivity, non-magnetic materials with high Fermi potential, Nucl. Instrum. Methods A 597(2-3), 222 (2008). 
[18] K. Green, P. G. Harris, P. Iaydjiev, D. J. R. May, J. M. Pendlebury, K. F. Smith, M. van der Grinten, P. Geltenbort and S. Ivanov, Performance of an atomic mercury magnetometer in the neutron EDM experiment, Nucl. Instrum. Methods A 404(2-3), 381 (1998).

[19] G. Ban et al., Demonstration of sensitivity increase in mercury free-spin-precession magnetometers due to laser-based readout for neutron electric dipole moment searches, Nuclear Instruments and Methods in Physics Research A 896, 129 (2018), doi:10.1016/j.nima.2018.04.025, 1804.05838.

[20] J. Pendlebury et al., Geometric-phase-induced false electric dipole moment signals for particles in traps, Phys. Rev. A 70, 032102 (2004), doi:10.1103/PhysRevA.70.032102.

[21] S. K. Lamoreaux and R. Golub, Detailed discussion of a linear electric field frequency shift induced in confined gases by a magnetic field gradient: Implications for neutron electricdipole-moment experiments, Phys. Rev. A 71(3), 032104 (2005).

[22] G. Pignol and S. Roccia, Electric-dipole-moment searches: Reexamination of frequency shifts for particles in traps, Phys. Rev. A 85(4), 042105 (2012).

[23] S. Afach et al., Measurement of a false electric dipole moment signal from ${ }^{199} \mathrm{Hg}$ atoms exposed to an inhomogeneous magnetic field, Eur. Phys. J. D 69(10), 225 (2015), doi:10.1140/epjd/e2015-60207-4, 1503.08651.

[24] G. Bison, B. Blau, M. Daum, L. Göltl, R. Henneck, K. Kirch, B. Lauss, D. Ries, P. Schmidt-Wellenburg and G. Zsigmond, Neutron optics of the PSI ultracold-neutron source: characterization and simulation, European Physical Journal A 56(2), 33 (2020), doi:10.1140/epja/s10050-020-00027-w, 1907.05730.

[25] C. Abel et al., Optically Pumped Cs Magnetometers Enabling a High-Sensitivity Search for the Neutron Electric Dipole Moment, Phys. Rev. A 101(5), 053419 (2020), doi:10.1103/PhysRevA.101.053419, 1912.04631.

[26] S. Afach et al., A device for simultaneous spin analysis of ultracold neutrons, Eur. Phys. J. A 51(11), 143 (2015), doi:10.1140/epja/i2015-15143-7, 1502.06876.

[27] G. Ban et al., Ultracold neutron detection with ${ }^{6}$ li-doped glass scintillators, Eur. Phys. J. A 52, 326 (2016).

[28] N. Ayres et al., Data Blinding for the nEDM Experiment at PSI (2019), 1912.09244.

[29] C. Abel et al., The n2EDM experiment at the Paul Scherrer Institute, EPJ Web Conf. 219, 02002 (2019), doi:10.1051/epjconf/201921902002, 1811.02340.

[30] G. Zsigmond, The mcucn simulation code for ultracold neutron physics, Nuclear Instruments and Methods in Physics Research Section A: Accelerators, Spectrometers, Detectors and Associated Equipment 881, 16 (2018), doi:https://doi.org/10.1016/j.nima.2017.10.065.

[31] S. Afach et al., Dynamic stabilization of the magnetic field surrounding the neutron electric dipole moment spectrometer at the Paul Scherrer Institute, J. Appl. Phys. 116, 084510 (2014), doi:10.1063/1.4894158, 1408.6752.

[32] M. Rawlik, A. Eggenberger, J. Krempel, C. Crawford, K. Kirch, F. M. Piegsa and G. QuÃCmÃ@)ner, A simple method of coil design, American Journal of Physics 86(8), 602 (2018), doi:10.1119/1.5042244. 
[33] Z. D. Grujić, P. A. Koss, G. Bison and A. Weis, A sensitive and accurate atomic magnetometer based on free spin precession, EPJD 69(5), 135 (2015), doi:10.1140/epjd/e2015-508753.

[34] G. Pignol, A magic magnetic field to measure the neutron electric dipole moment, Phys. Lett. B 793, 440 (2019), doi:10.1016/j.physletb.2019.05.014, 1812.01420.

[35] F. Piegsa, New Concept for a Neutron Electric Dipole Moment Search using a Pulsed Beam, Phys. Rev. C 88, 045502 (2013), doi:10.1103/PhysRevC.88.045502, 1309.1959.

[36] R. Picker, How the Minuscule Can Contribute to the Big Picture: The Neutron Electric Dipole Moment Project at TRIUMF, In Proceedings of the 14th International Conference on Meson-Nucleon Physics and the Structure of the Nucleon (MENU2016, p. 010005, doi:10.7566/JPSCP.13.010005 (2017), 1612.00875.

[37] T. Ito et al., Performance of the upgraded ultracold neutron source at Los Alamos National Laboratory and its implication for a possible neutron electric dipole moment experiment, Phys. Rev. C 97(1), 012501 (2018), doi:10.1103/PhysRevC.97.012501, 1710.05182.

[38] M. Ahmed et al., A New Cryogenic Apparatus to Search for the Neutron Electric Dipole Moment, JINST 14(11), P11017 (2019), doi:10.1088/1748-0221/14/11/P11017, 1908.09937.

[39] D. Wurm, D. H. Beck, T. Chupp, S. Degenkolb, K. Fierlinger, P. Fierlinger, H. Filter, S. Ivanov, C. Klau, M. Kreuz, E. Lelièvre-Berna, T. Lins et al., The PanEDM neutron electric dipole moment experiment at the ILL, In European Physical Journal Web of Conferences, vol. 219 of European Physical Journal Web of Conferences, p. 02006, doi:10.1051/epjconf/201921902006 (2019). 